\title{
Practice of prophylactic antibiotic and timing of administration in two common elective surgeries in Pakistan: An audit-based study
}

\author{
Original \\ Zakir Khan', Naveed Ahmed', Asim-u-Rehman', Faiz Ullah Khan', \\ Maria Auxiliadora Parreiras Martins ${ }^{3}$. \\ Article \\ ${ }^{1}$ Department of Pharmacy, Quaid-i-Azam University Islamabad, Pakistan. \\ ${ }^{2}$ Department of Pharmacy, Administration and Clinical Pharmacy, School of Pharmacy, \\ Health Science Centre, Xi'an Jiaotong University, China. \\ ${ }^{3}$ Faculdade de Farmácia, Universidade Federal de Minas Gerais, Brazil
}

\begin{abstract}
Antibiotic prophylaxis usage is one of the main actions to prevent and reduce rate of surgical site infections. Antimicrobial stewardship programme is also focused on audit of surgical antibiotic prophylaxis (SAP). The objective of study was to evaluate the appropriateness of SAP and their pattern of utilization in two tertiary-care teaching hospitals in Islamabad (Pakistan). A total of 965 elective surgeries were performed during the 9 months study period. The two most common elective surgical procedures were Laparoscopic cholecystectomy and Direct right inguinal hernia, that were performed on 443 patients. Adherence to the Standard International Guidelines (CDC, 2017) about appropriate use of antibiotic and timing of administration were main outcomes. The mean age of patients was $43.5 \pm 16.3$ years. SAP was appropriate according to guidelines in only $5 \%(n=22)$ of cases. Appropriate use of SAP was greater in direct right inguinal hernia $(10 \%)$ than laparoscopic cholecystectomy $(1.2 \%)$ surgery; $\mathrm{P}=0.001$. The drug of choice cefazolin was only prescribed to $4.2 \%$ ( 19 out of 443) of patients. Timing of administration was appropriate in 50.8\% (225 out of 443) of the procedures. Compliance with timing was significantly lower in Hospital GH (32\%) as compared to Hospital PH (71\%); P $<0.001$. Length of stay was significantly different $(\mathrm{P}=0.001)$ between surgical procedures. The most common antibiotics used inappropriately were ceftriaxone and Cefuroxime. Present study found an overall low adherence to SAP mainly regarding choice and timing of administration. Educational intervention and urgent need for implementation of antimicrobial stewardship programme are required.
\end{abstract}

Received: 22 June 2019, Accepted: 29 June 2019

Key Words: Antibiotic; compliance; hospitals; infections, laparoscopic cholecystectomy; .

Corresponding Author: Zakir Khan, MSc, Department of Pharmacy, Quaid-i-Azam University, Islamabad, Pakistan, Tel.: +92 3486062557, E-mail: zakirkhan300@gmail.com

Bulletin of Faculty of Pharmacy, Cairo University, ISSN: 1110-0931, Vol. 57, No. 1

\section{INTRODUCTION}

Surgical site infections (SSIs) are among the most common hospital-acquired infections ${ }^{[1,2]}$. SSIs are implicated in one-third of postoperative deaths and accounts for $8 \%$ of all deaths caused by hospital-acquired infections. The burden of SSIs in low and middle-income countries (LMICs) is growing ${ }^{[3]}$. A global research project was carried out globally in 2015, including 10, 475 patients from 58 countries. It showed that the incidence of SSI was more than doubled in low-income countries $(20.0 \%)$ as compared to high income countries (7.4\%). Dirty surgeries performed were much higher in low-income countries ( $29.7 \%$ vs $16.6 \%$ in high-income countries), which was in turn associated with a very high rate of SSI $(34.5 \%$ low income vs $15.4 \%$ high income $)^{[3,4]}$. Furthermore, SSIs cause pain and discomfort, increase hospital stay and a greater risk of secondary infectious complications. This has an important economic impact with an attributable cost in the UK of $£ 30$ million per year ${ }^{[3,5]}$.

Centers for Medicare and Medicaid Services Surgical Care Improvement Project (SCIP) majorly focus on prevention of SSI. That is a national priority for Pakistan ${ }^{[6]}$. SSIs are an important public health threat that concerns the safety of patients and health care professionals ${ }^{[2,7]}$. These are one of the leading causes of morbidity and mortality in low- and middle-income countries. Higher incidence of SSI is also associated with higher costs of medications ${ }^{[2,8]}$.

Laboratory studies in the early 1960s established principles of antibiotic prophylaxis against SSIs ${ }^{[9]}$. Surgical antibiotic prophylaxis (SAP) is among the safest ways to reduce SSIs. The timing of administration and selection of antibiotics are important measures for the prevention of SSIs $^{[1,10,11]}$. Despite this evidence, the recommendations are not routinely followed. Antibiotics have been reported to 
be used excessively and inappropriately for the prevention of SSIs in surgical patients ${ }^{[7]}$. Non-compliance with timing and inappropriate choice of antibiotic increase the risk of SSIs and resistance respectively ${ }^{[12,13]}$. Thus, the appropriate use of these agents is a critical issue for patient ${ }^{[8,14]}$.

Appropriateness of the prophylactic antibiotics use in clinical settings has been addressed by various epidemiological studies in different countries ${ }^{[2,7,8,14]}$. However, limited data is published on this topic in Pakistan. Our country has a tropical climate, which poses a significant challenge clinically for the management of a wide variety of infections. Therefore, the present study was conducted to report surgical antibiotic prophylaxis (SAP) use and adherence with standard guidelines in two most common elective surgical procedures in Pakistan.

\section{MATERIAL AND METHODS}

\subsection{Study design and Setting}

A cross-sectional observational study was conducted between August 20, 2017 and April 20, 2018. General surgery departments of Pakistan Institute of Medical Science (PIMS) which is government hospital (GH) and Shifa International Hospital (SIH), a private hospital (PH) at Islamabad, Pakistan were selected. GH is a 600 beds tertiary care hospital and one of the region's leading tertiary level hospitals which includes 22 medical and surgical specialist centers. $\mathrm{PH}$ is also a tertiary care, multi-specialty 500 beds teaching hospital. Both hospitals provide medical facilities to the Rawalpindi and Islamabad regions. These are a national level referral hospital for Northern areas of Azad Jammu Kashmir, Khyber Pakhtunkhwa and Punjab, Pakistan. Therefore, it is approached by a population of different regions of Pakistan and more indicative of the country's health status.

\subsection{Study Population}

Adults patients ages greater than 18 years and with no previous infection and surgery were included. A total of 965 patients were subjected to elective surgical procedures during study period. Among them laparoscopic cholecystectomy (Lap-Chole) and direct right inguinal hernia (DRIH) were performed in 504 patients. A total of 61 of $504(12 \%)$ patients were excluded due to either incomplete medical record (33 patients) or having age less than 18 years (28 patients). Finally, 443 (Lap-Chole $n=250$, DRIH $n=193$ ) cases were recruited for present study. These are frequent surgeries in selected hospitals, and they represent procedures generally classified as cleancontaminated and clean.

\subsection{Data Collection Method}

Each patient was requested for participation and a written informed consent was obtained from every patient after explaining the study objectives. The medical record of each patient was reviewed, and summarized on a standardized case report form. The following characteristics were collected from each patient undergoing surgery: age, gender, weight, height, admission diagnosis, type and duration of the surgical intervention, details of antibiotic prophylaxis including type of antibiotic agents, administration route, dosage, time, and length of hospital stay at time of survey.

\subsection{Outcomes measures}

The SAP were judged as has been judged appropriate if the antibiotic and the timing were in accordance with the Centers for disease control (CDC) and prevention guideline for the prevention of surgical site infection, 2017 ${ }^{[10]}$. WHO Anatomical Therapeutic Chemical (ATC) classification system was used for most common classes and combination of antibiotics ${ }^{[15]}$. For each surgical procedure the following items were considered: type of antibiotic and the timing of its administration within 60 minutes before surgical incision. Present study assessed different aspect of pre-operative antibiotic administration. These aspects were supported by Strength of Evidence A which include level 1, level 2 and level 3 evidences. Level 1 includes evidence from large, well conducted, randomized controlled clinical trials or meta-analysis, Level 2 from small, well conducted randomized controlled clinical trials and level 3 include well conducted cohort studies. According to protocols, patients undergoing LapChole and DRIH procedures, a single dose of cefazolin is sufficient as antibiotic prophylaxis. Clindamycin or vancomycin is an acceptable alternative in patients with a documented $\beta$-lactam allergy ${ }^{[1,10]}$. Details are summarized in (Table 1).

Table 1: Summary of compliance criteria with Standard treatment Guidelines $^{[1,10]}$

\begin{tabular}{clccc}
\hline Pre-operative Prescription & & & \\
\hline Procedures & $\begin{array}{l}\text { Antibiotic use } \\
\text { and route }\end{array}$ & Dose & $\begin{array}{c}\text { Timing } \\
\text { (min) }\end{array}$ & $\begin{array}{c}\text { Strength of } \\
\text { Evidence }\end{array}$ \\
\hline $\begin{array}{c}\text { Lap-Chole } \\
\text { First line }\end{array}$ & Cefazolin IV & $2 \mathrm{~g}$ & 60 & $\mathrm{~A}$ \\
Alternative & Clindamycin IV & $900 \mathrm{mg}$ & 60 & $\mathrm{~A}$ \\
& Gentamicin IV & $5 \mathrm{mg} / \mathrm{kg}$ & 60 & $\mathrm{~A}$ \\
DRIH & & & & \\
First line & Cefazolin IV & $2 \mathrm{~g}$ & 60 & $\mathrm{~A}$ \\
Alternative & Clindamycin IV & $900 \mathrm{mg}$ & 60 & $\mathrm{~A}$ \\
& Vancomycin & $15 \mathrm{mg} / \mathrm{kg}$ & 120 & $\mathrm{~A}$ \\
\hline
\end{tabular}

\subsection{Sample size}

To determine the number of surgical procedures needed to sufficiently power the analysis, it was expected overall appropriate use of prophylactic antibiotics to be approximately $50 \%$, assuming a confidence interval of $95 \%$, a tolerable level of type- 1 error of $5 \%$. The minimum size required of the sample was estimated to be at least 384 .

\subsection{Statistical Analysis}

The outcomes of interest were the overall adherence with guidelines on appropriateness of antibiotic choice and timing of antibiotic administration prior to surgery amongst surgical patients. Descriptive statistics was used for mean, 
range, frequency, percentage and standard deviation. Association between compliance of antibiotic choice and timing was determined using Pearson Chi Square. Phi and Cramer's V test were also used to show strength of association among variables. All tests were two-tailed and a p-value of 0.05 or less was defined as statistically significant. The following independent variables were included: gender (male $=0$, female $=1$ ), age (continuous, in years), weight (continuous, in kilogram), surgery type (Lap-Chole=1, DRIH=2), antibiotic name (continuous nominal), dose (continuous, in gram), administration time of prophylactic antibiotics (in hours-minutes), start time of surgery (in hours-minutes), time of PPA administration before surgical incision (continuous, in minutes), end time of surgery (in hours-minutes), duration of stay (continuous, in days), hospital type (GH (government hospital) $=0, \mathrm{PH}$ (private hospital) $=1$ ), antibiotic use (inappropriate choice $=$ 0 , appropriate choice $=1$ ). These variables and comparisons are based on previous study ${ }^{[7]}$. Statistical analysis was performed using Statistical Package for Social Sciences (SPSS Version 22.0).

\section{Ethics approval}

As a first step, approvals were obtained from bioethical committee of Quaid-i-Azam University, Islamabad, Pakistan (No. DFBS/2017-623) and from the Ethical/ Institutional review board of Pakistan Institute of Medical Sciences (No. F.1-1/2017/ERB/SZABMU/) and Shifa international hospital (No. IRB-637-085-2017), Islamabad, Pakistan. A written and oral informed consent was also taken from all participants before observing medication records.

\section{RESULTS}

Among the 443 patients, most were men (278; $62.7 \%$ ) with an average age of 43.5 years (18-98). Sample descriptions are listed in (Figure 1) and general characteristics of patients are given in (Table 2).

Table 2: The general characteristics of patients in two common elective surgeries

\begin{tabular}{|c|c|c|c|c|}
\hline Characteristics & Lap-Chole & DRIH & Total sample & P-value \\
\hline Hospital type (GH/PH) n (\%) & $123 / 127(49.2 / 50.8)$ & $109 / 84(56.5 / 43.5)$ & $232 / 211(52.4 .6 / 47.6)$ & \\
\hline Number of patients $n(\%)$ & $250(56.4)$ & $193(43.6)$ & $443(100 \%)$ & 0.001 \\
\hline Gender Male/Female n (\%) & $131 / 119(52.4 / 47.6)$ & $147 / 46(76.1 / 23.9)$ & $278 / 165(62.7 / 37.3)$ & 0.001 \\
\hline Age, year & $45.75 \pm 16.6(18-92)$ & $40.58 \pm 15.6(18-98)$ & $43.50 \pm 16.37(18-98)$ & .091 \\
\hline \multicolumn{5}{|l|}{ Mean \pm standard deviation (range) } \\
\hline Weight, $\mathrm{Kg}$ & $75.7 \pm 10.5(53-107)$ & $73.9 \pm 9.8(45-105)$ & $74.97 \pm 10.25(45-107)$ & .205 \\
\hline \multicolumn{5}{|l|}{ Mean \pm standard deviation (range) } \\
\hline Length of stay & $3.2 \pm .60(2-4)$ & $2.6 \pm .54(2-4)$ & $2.99 \pm .661(2-4)$ & 0.001 \\
\hline Mean \pm standard deviation (range) & & & & \\
\hline
\end{tabular}

Legends: n number, \% percentage, Lap-Chole Laparoscopic cholecystectomy, DRIH Direct Right Inguinal Hernia, GH Government hospital, PH Private hospital 


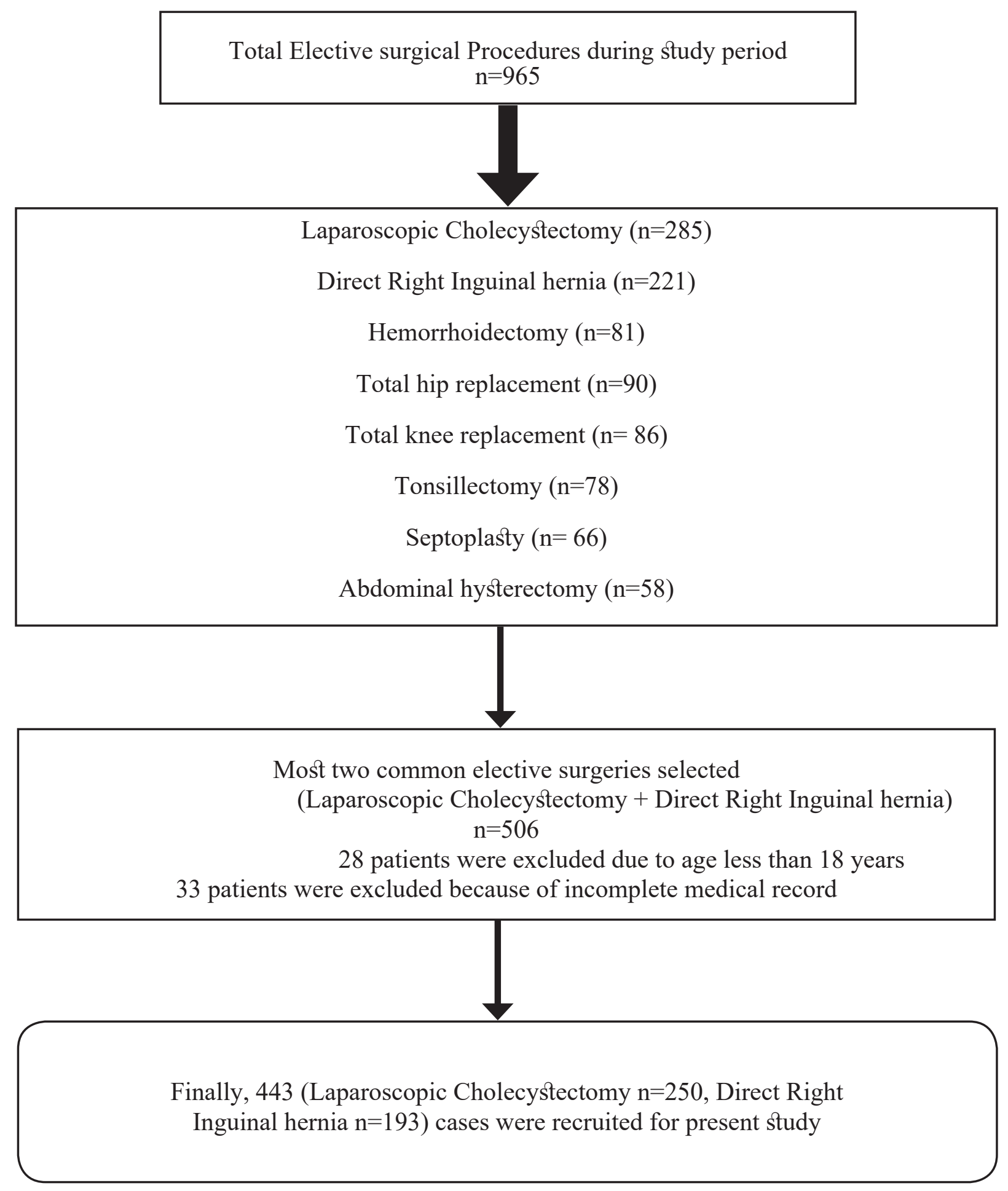

Fig. 1: Flowchart for inclusion of eligible two common surgeries 
Antibiotic prophylaxis was appropriate only in 5\% of the procedures. Compliance with antibiotic prophylaxis was not significantly different in Hospital GH (4\%) and Hospital PH $(6 \%) ; P=0.270$. Phi and Cramer's V test value was .052 which shows lower strength of association among variables. Appropriate use of antibiotics was greater in DRIH (10\%) as compared to Lap-Chole (1.2\%) surgery; $P=0.001$. Phi and Cramer's V test value was .197 which ultimately shows strong association among variables. The evaluation of the appropriateness of the timing of prophylactic administration of antibiotics, defined as only an injection occurred within 60 minutes before surgical incision (only for vancomycin within 120 minutes) ${ }^{[1,10,16]}$ indicates an appropriateness only in $50.8 \%$ (225 out of 443 ) of the surgical procedures. Timing of antibiotic prophylaxis was inappropriate in more than $49 \%$ of the procedures. Compliance with timing was significantly lower in Hospital GH (32\%) compared to Hospital PH $(71 \%) ; P<0.001$ (Phi and Cramer's V test value $=.387$ ) Whereas, there was no significant difference between DRIH $(49 \%)$ and Lap-chole $(52 \%)$ surgeries regarding timing of antibiotic administration; $\mathrm{P}=0.562$ (Phi and Cramer's V test value $=.028$ ). The details are reported in (Table 3 ).

Table 3: Compliance with the International guidelines on antibiotic prophylaxis

\begin{tabular}{|c|c|c|c|c|}
\hline \multirow{2}{*}{$\frac{\text { Variables }}{\mathrm{n}(\%)}$} & \multicolumn{2}{|c|}{ Appropriate antibiotic prophylaxis } & \multicolumn{2}{|c|}{ Appropriate timing of antibiotic administration } \\
\hline & Yes n (\%) & No n $(\%)$ & Yes n (\%) & No n $(\%)$ \\
\hline Gender Male 278 (62.7) & $17(6.1)$ & $261(93.9)$ & $143(51.4)$ & $135(49.6)$ \\
\hline Females $165(37.3)$ & $5(3)$ & $160(97)$ & $82(49.7)$ & $83(50.3)$ \\
\hline $\begin{array}{l}\text { Length of stay (days) } \\
2 \text { days - } 99(22.3)\end{array}$ & $9(9)$ & $90(91)$ & $56(56.6)$ & $43(43.4)$ \\
\hline 3 days- $250(56.4)$ & $12(4.8)$ & $238(95.2)$ & $133(53.2)$ & $117(46.8)$ \\
\hline 4 days - 94 (21.2) & $1(1)$ & $93(99)$ & $36(38.3)$ & $58(61.7)$ \\
\hline Surgery type Lap-Chole 250 (56.4) & $3(1.2)$ & $247(98.8)$ & $130(52)$ & $120(48)$ \\
\hline DRIH 193 (43.6) & $19(10)$ & $174(92)$ & $95(49.2)$ & $98(50.8)$ \\
\hline Hospital Type GH 232 (52.4) & $9(4)$ & $223(96)$ & $75(32.3)$ & $157(67.7)$ \\
\hline PH 211 (47.6) & $13(6)$ & $198(94)$ & $150(71)$ & $61(28.9)$ \\
\hline Total n (\%) & $22(5)$ & $421(95)$ & $225(50.8)$ & $218(49.2)$ \\
\hline
\end{tabular}

Legends: $n$ number, \% percentage, Lap-Chole Laparoscopic cholecystectomy, DRIH Direct Right Inguinal Hernia, GH Government hospital, PH Private hospital

Prophylaxis antibiotics were administered in $100 \%$ of the procedures. The most common antibiotics were ceftriaxone $70.6 \%(313 / 443)$, cefuroxime $9.5 \%$ and azithromycin $4 \%$. Most common combination of antibiotic was cefoperazone plus sulbactam $4 \%$. The type of antibiotic administered was appropriate only in 22 cases (4.9\%), and the antibiotics most frequently used inappropriately were ceftriaxone, cefuroxime, and amoxicillin plus clavulaunic acid. Details on prescribed antibiotics are listed in (Table 4).

Table 4: Frequency and percentages of various surgical antibiotic prophylaxis prescribed

\begin{tabular}{|c|c|c|c|c|c|}
\hline \multirow{2}{*}{ Antibiotics (dose) } & \multirow{2}{*}{ WHO/ATC code } & \multicolumn{2}{|c|}{ Lap-chole } & \multicolumn{2}{|c|}{ DRIH } \\
\hline & & $\mathrm{n}$ & $\%$ & $\mathrm{n}$ & $\%$ \\
\hline Ceftriaxone (2g) & J01DD04 & 206 & 82 & 107 & $55.4 \%$ \\
\hline Cefoperazone+Sulbactam (1g) & J01DD62 & 18 & 7.2 & - & - \\
\hline Cefuroxime $(1.5 \mathrm{~g})$ & J01DC02 & 7 & 2.8 & 35 & 18.1 \\
\hline Amoxicillin+Clavulaunic acid (1.2 g) & J01CR02 & 6 & 2.4 & 11 & 5.7 \\
\hline Ciprofloxacin (500 mg) & J01MA02 & 6 & 2.4 & - & - \\
\hline *Cefazolin $(2 \mathrm{~g})$ & J01DB04 & 2 & 0.8 & 17 & 8.8 \\
\hline Piperacillin Sulbactam (4.5g) & J01CR05 & 4 & 1.6 & 1 & 0.5 \\
\hline$* *$ Vancomycin $(500 \mathrm{mg})$ & J01XA01 & 1 & 0.4 & 2 & 1 \\
\hline Azithromycin (500 mg) & J01FA10 & - & - & 18 & 9.3 \\
\hline Amikacin (500 mg) & J01GB06 & - & - & 2 & 1 \\
\hline Total & & 250 & 100 & 193 & 100 \\
\hline
\end{tabular}

Legends: GH Government hospital, PH Private hospital, n Number, \% percentage, WHO/ATC World Health Organization/Anatomical Therapeutic Classification, * First Choice, ** Second Choice 


\section{DISCUSSION}

This study is a comprehensive assessment of appropriate prescription of SAP amongst the most common surgical procedures in two tertiary care hospitals. This kind of research has not been previously conducted in Pakistan. We found a substantial proportion of inappropriate SAP contradictory with evidence based standard treatment guidelines. Appropriate choice of antibiotic is important step in surgical prophylaxis. Selection of antibiotic in current study was not adherent with the standard criteria. The SAP was administered in line with standard international recommendations in less than $5 \%$ of patients. These findings are aligned with other studies conducted in Italy ${ }^{[17]}$ and Brazilian hospital ${ }^{[18]}$ which reported adherence rate of $5.7 \%$ and $3-5.8 \%$ respectively. This value is lower than other studies conducted in Italy $18.1 \%{ }^{[7]}$, France $19.4 \%^{[19]}$, India $52 \%{ }^{[20]}$ and Germany $70.7 \%^{[21]}$, which observed more adherence rate accordance to guidelines in surgical patients. The first step for the appropriate use of SAP is to provide educational training on antibiotic stewardship programme and associated SSIs risk. Many previously conducted studies demonstrated the benefits of educational intervention for antibiotic prophylaxis. Different studies conducted in Nigeria ${ }^{[12]}$, Italy ${ }^{[17]}$ and Australia ${ }^{[22]}$ found that compliance to antibiotic prophylaxis guidelines improved with increased awareness among surgeons and other health care team members.

Appropriate timing of administration of SAP was $50.8 \%$ in present study. These findings are align with study conducted in Italy which showed $53.4 \%$ adherence rate with guidelines ${ }^{[7]}$. Previous studies conducted in Australia $(43.3 \%)^{[22]}$, Northern Nigeria $(16.5 \%)^{[12]}$ and Egypt $(5 \%)^{[23]}$ reported low adherence rate as compared to our study. Whereas, higher rate was reported by other studies conducted in Greece $(100 \%)^{[14]}$, England $(86.4 \%)^{[24]}$, France $(76.6 \%)^{[19]}$ and Italy $(75.7 \%)^{[25]}$. It is a best evidence that delayed administration of SAP is associated with 2 times greater risk of SSIs as compared to timely administration ${ }^{[12]}$. Lack of standard guidelines and protocols for antibiotic prophylaxis in these hospitals could be a reason of non-compliance in our study. Similar reason was also coded in previous studies ${ }^{[12,23,26]}$. Lack of knowledge, unavailability of clinical pharmacist and poor collaboration with health care team are another reason for non-compliance in present study. Further, large scale and multi-centered studies are needed to dig out others contributing factors of non-compliance.

In the present study the most common antibiotics were ceftriaxone. A study conducted in Ethiopia also reported that ceftriaxone was excessively and inappropriately used in their settings ${ }^{[27]}$. These finding are also aligned with the result of a systematic review which, reported third generation cephalosporins as a major type of noncompliant antibiotic ${ }^{[28]}$. Whereas, these findings are deviated from previously conducted studies in Singapore, Greece, Germany and Italy which showed most common antibiotics were cefazolin, ceforanide, cefuroxime and levofloxacin respectively ${ }^{[7,8,14,21]}$. The selected antibiotics for surgical prophylaxis should have coverage against pathogens, be less toxic and inexpensive ${ }^{[1,10,29]}$. Cefazolin should be used for surgical prophylaxis except in cases such as significant beta-lactam allergy, known MRSA colonization, or surgical sites with probable organisms that are not covered by cefazolin alone (e.g., appendectomy, colorectal). Clindamycin or vancomycin are often used as alternatives in those with significant beta-lactam allergies $^{[1,6,10]}$.

There are some potential limitations that should be noted. First, the design of the study implicates that adherence to prescribing guideline was only considered when it was recorded in the patients' medical charts. Before and after assessment was not done, further intervention required in future studies. Second, the study results may reflect the epidemiology and guideline adherence in only two common surgical elective surgical procedures across hospitals. However, the goals of the study were to provide an overview of the antibiotic prophylaxis guideline adherence and the appropriateness of prescribed prophylaxis among all patients undergoing surgical treatment, and we do believe that our data provided insights into daily clinical practice. Third, the reasons for non-adherence to antibiotic prophylaxis guidelines were beyond the scope of the current study. Moreover, the present study used published recommendations of Centers for Disease Control and Prevention guideline for the prevention of surgical site infection, 2017, since, there was no local consensus guidelines in selected hospitals. Despite the limitations, as to the best of our knowledge, no previous studies have been focused on extensive audit of prevalent practice of prophylactic antibiotic prescribing behavior in selected two common elective surgeries, in a global or local perspective. Therefore, these data are highly important because they provide information that contributes to the understanding of the appropriateness of the prescription of antibiotics prophylaxis prior to surgery and the pattern of antibiotic amongst surgical patients.

\section{CONCLUSION}

Present study found an overall low adherence to antibiotic prophylaxis guidelines regarding SAP choice and timing of administration. The results provide evidence that health care providers should be aware of their larger role in reducing unnecessary and inappropriate prescription of antibiotics prophylaxis in patients prior to surgery. Compliance with guidelines by surgeons is remains a challenge, as reported by previously conducted studies around the globe and also in present study. Real actions are urgently needed for the implementation of guidelines. There is a clear need for additional efforts and educative interventions to improve antibiotic prophylaxis which is a basic part of antibiotic stewardship programme.

\section{ACKNOWLEDGMENTS}

Special thanks to the staff of selected hospitals for their assistance during data collection. 


\section{CONFLICTS OF INTEREST}

There are no conflicts of interest.

\section{REFERENCES}

1. D.W. Bratzler, et al. Clinical practice guidelines for antimicrobial prophylaxis in surgery, Surgical infections. 14 (2013) 73-156.

2. S.A. Khan, et al. Survey and evaluation of antibiotic prophylaxis usage in surgery wards of tertiary level institution before and after the implementation of clinical guidelines, Indian J Surg. 68 (2006) 3-9.

3. Collaborative, Determining the worldwide epidemiology of surgical site infections after gastrointestinal resection surgery: protocol for a multicentre, international, prospective cohort study (GlobalSurg 2), BMJ open.7 ( 2017) e012150.

4. S. Earnshaw, et al. Global collaboration to encourage prudent antibiotic use, Lancet Infect Dis, 13 (2013) 1003-1004.

5. J. Tanner, et al. Post-discharge surveillance to identify colorectal surgical site infection rates and related costs, J Hosp Infect. 72 (2009) 243-250.

6. D.W. Bratzler, D.R. Hunt, The surgical infection prevention and surgical care improvement projects: national initiatives to improve outcomes for patients having surgery, Clin Infect Dis. 43 ( 20063) 322-330.

7. F. Napolitano, et al. Evaluation of the appropriate perioperative antibiotic prophylaxis in Italy, PloS one. 8 (2013) e79532.

8. B. Young, et al. Non-concordance with Surgical Site Infection Prevention Guidelines and the Rate of Surgical Site Infections for General Surgical, Neurological and Orthopedic Procedures, Antimicrob. Agents Chemother. 4 (2011) 562-11.

9. J.F Burke, The effective period of preventive antibiotic action in experimental incisions and dermal lesions, Surgery. 50 (1961) 161-168.

10. S.I. Berríos-Torres, et al. Centers for disease control and prevention guideline for the prevention of surgical site infection, JAMA surgery. 152 (2017) 784-791

11. A.J Mangram, et al. Guideline for prevention of surgical site infection, Infect Control Hosp Epidemiol. 20 (1999) 247-280.

12. U. Abubakar, S. Sulaiman, A. Adesiyun, Utilization of surgical antibiotic prophylaxis for obstetrics and gynaecology surgeries in Northern Nigeria, Int J Clin Pharm. 40 (2018) 1037-1043.

13. S.W. de Jonge, et al. Timing of preoperative antibiotic prophylaxis in 54,552 patients and the risk of surgical site infection: a systematic review and meta-analysis, Medicine. 96 (2017) 29-35.

14. C. Tourmousoglou, et al. Adherence to guidelines for antibiotic prophylaxis in general surgery: a critical appraisal, J Antimicrob Chemother. 61 (2007) 214-218.

15. World Health organization, The anatomical therapeutic chemical classification system with defined daily doses (ATC/DDD) Methodology. https://www.who.int/medicines/regulation/ medicines-safety/toolkit methodology/en/>, 2018 (accessed 14.12.18).

16. A.S.H.P Pharmacists, American Society of HealthSystem Pharmacists therapeutic guidelines on antimicrobial prophylaxis in surgery, Am J Health Syst Pharm. 56 (1999) 1839-1888.

17. M.L Giordano, Squillace, M. Pavia, Appropriateness of surgical antibiotic prophylaxis in pediatric patients in Italy, Infect Control Hosp Epidemiol. 38 (2017) 823-831.

18. C. Schmitt, et al. Applying validated quality indicators to surgical antibiotic prophylaxis in a Brazilian hospital: learning what should be learned, Am J Infect Control. 40 (2012) 960-962.

19. K. Miliani, et al. Non-compliance with recommendations for the practice of antibiotic prophylaxis and risk of surgical site infection: results of a multilevel analysis from the INCISO Surveillance Network, J Antimicrob Chemother. 64 (2009) 1307-1315.

20. L. Parulekar, et al. How good is compliance with surgical antibiotic prophylaxis guidelines in a tertiary care private hospital in India? A prospective study, Indian J Surg. 71 (2009) 15-18.

21. C. Hohmann, et al. Adherence to guidelines for antibiotic prophylaxis in surgery patients in German hospitals: a multicentre evaluation involving pharmacy interns, Infection. 40 (2012): p. 131-137.

22. S. Jaber, et al. Appropriateness of surgical antibiotic prophylaxis for breast surgery procedures, Int $\mathrm{J}$ Clin Pharm. 39 (2017) 483-486.

23. T. Saied, et al. Antimicrobial stewardship to optimize the use of antimicrobials for surgical prophylaxis in Egypt: a multicenter pilot intervention study, Am J Infect Control. 43 (2015) 67-71.

24. M.T. Hawn, et al. Association of timely administration of prophylactic antibiotics for major surgical procedures and surgical site infection, J Am Coll Surg. 206 (2008) 814-819. 
25. P. Durando, et al. Adherence to international and national recommendations for the prevention of surgical site infections in Italy: results from an observational prospective study in elective surgery, Am J Infect Control. 40 (2012) 969-972.

26. M. Askarian, et al. Adherence to American Society of Health-System Pharmacists surgical antibiotic prophylaxis guidelines in Iran, Infect Control Hosp Epidemiol. 27(2006) 876-878.

27. G. Alemkere, Antibiotic usage in surgical prophylaxis: A prospective observational study in the surgical ward of Nekemte referral hospital, PloS one. 6 (2018) e0203523.

28. R.S Ng, C.P. Chong, Surgeons' adherence to guidelines for surgical antimicrobial prophylaxis-a review, Australas Med J. 5 (2012)534-39.

29. W.P. Safety, WHO guidelines for safe surgery saves lives., Geneva: World Health Organization. https://apps.who.int/iris/bitstream/ handle/10665/44185/9789241598552_eng.pdf;jse ssionid=A1095AA73CC 79565451555725 DAC02 13 ? sequence $=1.2009$ (acessed 01-01-19). 